\title{
Surgical treatment for lumbar spinal stenosis: a single-blinded randomized controlled trial
}

\author{
Luiz Claudio Lacerda Rodrigues ${ }^{1,2}$ and Jamil Natour ${ }^{1 *}$ (D)
}

\begin{abstract}
Objective: To evaluate the effectiveness of surgery for the management of patients with symptomatic lumbar spinal stenosis.

Methods: Sixty-three patients with lumbar canal stenosis were randomized into two groups: the intervention group (IG) and control group (CG). IG patients underwent surgery and both groups participated in the same physical therapy program twice a week for a period of 12 weeks and were followed up at 1 year. The primary endpoint was visual analogue scale for pain, and the secondary endpoints were function (6-min walk test, Roland Morris and Oswestry questionnaires), quality of life (SF-36 questionnaire) and satisfaction with treatment (Likert scale).

Results: No significant difference between groups was observed for pain over time $(p=0.145)$. Significant differences between groups, in favor of the IG, were observed for the Oswestry score $(p=0.006)$ and vitality domain score of the SF-36 $(p=0.047)$. Function in the Roland Morris and 6-min walk test and the role of the physical domain of SF-36 also showed significant differences between the groups; however, these differences occurred due to a worsening of the IG in the short term, and the medium-term. The Likert scale demonstrated greater satisfaction with the IG treatment compared to control group.
\end{abstract}

Conclusions: Lumbar stenosis surgery did not improve pain in short and medium terms. Function and vitality were better in the group that underwent surgery in the medium term, and patients were more satisfied with the surgical treatment.

Trial registration: Clinicaltrials.gov (NCT02879461).

Keywords: Lumbar stenosis, Surgery, Back pain, Function, Spine, Quality of life

\section{Introduction}

Lumbar canal stenosis was first described by Antoine Portal in 1803. However, the first association of changes in the diameter of the spinal canal with clinical features and neurogenic claudication was performed by Verbiest $[1,2]$.

Neurogenic claudication, characterized by low back pain or weakness in the lower limbs, progressively worsening with an orthostatic position or walking, and improving

\footnotetext{
* Correspondence: jnatour@unifesp.br

${ }^{1}$ Rheumatology Division, Universidade Federal de São Paulo - Escola Paulista de Medicina (Unifesp-EPM), Sao Paulo, SP 04023900, Brazil

Full list of author information is available at the end of the article
}

after stopping, sitting or tilting the body forward, is the most common symptom of lumbar canal stenosis. The less common symptom is, unilateral radiculopathy [3].

Lumbar canal stenosis can be confused with many diseases, such as vascular claudication, tumors, peripheral neuropathy and knee or hip osteoarthritis [4]. The diagnosis is clinical, accompanied by findings in the imaging tests that confirm the narrowing of the spine canal [5].

Lumbar canal stenosis is among the most common causes of spinal surgery in individuals over 65 years. Functional disability and pain lead many patients to surgery after insufficient results are obtained with clinical

(c) The Author(s). 2021 Open Access This article is licensed under a Creative Commons Attribution 4.0 International License, which permits use, sharing, adaptation, distribution and reproduction in any medium or format, as long as you give appropriate credit to the original author(s) and the source, provide a link to the Creative Commons licence, and indicate if changes were made. The images or other third party material in this article are included in the article's Creative Commons licence, unless indicated otherwise in a credit line to the material. If material is not included in the article's Creative Commons licence and your intended use is not permitted by statutory regulation or exceeds the permitted use, you will need to obtain permission directly from the copyright holder. To view a copy of this licence, visit http://creativecommons.org/licenses/by/4.0/. 
treatment. Even before adequate clinical treatment, many patients appeal to surgery, believing that it is a definitive a solution [6].

This is a large surgery, often involving laminectomy, arthrodesis and instrumentation, with a high cost to the health system.

The scientific literature, however, fails to demonstrate the advantages of surgical treatment. There are studies of poor methodological quality and those generally comparing different surgical techniques and not comparing surgical treatment with clinical treatment [7-11].

The aim of this study was to determine the effectiveness of surgery in the management of patients with symptomatic lumbar spinal stenosis.

\section{Methods}

A single-blind parallel randomized controlled trial was conducted with a 48-week follow-up period. Sixty-three patients of both genders, between 50 and 75 years old, were selected from the outpatient clinic and randomized into intervention group (IG) or control group (CG) using electronic randomization. Opaque envelopes were used to keep the secret of allocation. The study was approved by the institution's ethics committee and registered in clinicaltrials.gov (NCT02879461).

All the procedures were in accordance with the ethical standards of the responsible committee on human experimentation and with the Declaration of Helsinki and all patients agreed to participate and signed the informed consent form.

Only patients with up to three vertebral levels of lumbar canal stenosis were included in the study. The clinical diagnosis included the presence of claudication with less than $100 \mathrm{~m}$ of walking and at least two of the following complaints in the lower limbs: pain, weakness, numbness, and tingling that worsen with gait and improve with rest, whether they are associated with low back pain for at least 6 months [12].

The presence of lumbar canal stenosis was confirmed when the lumbar canal area was smaller than $100 \mathrm{~mm}^{2}$, based on the Hamanishi criteria [13], measured on the lumbar spine MRI following L3 to S1, performed in a 1 Tesla device (Philips, Giroscan). The examination was performed with the patient in dorsal decubitus and with cushion on the knees to maintain flexion of the hip and knees. To avoid interference in the measurements of spinal canal due to angulation, all cuts were performed parallel to the discs, accepting an angulation difference of at most $5^{\circ}[14]$.

The spinal canal diameter measurement was based on the laterolateral (a) and anteroposterior (b) diameter, always on the largest axis, with data provided by a computer program (OSIRIX $^{\circ}$ 2010) in the scanned image. These values were divided by two, individually, and multiplied by the value of $\pi$ (PI) $[3,15]$. The result obtained was multiplied by a constant that varied between 0.8 (when the spinal canal was circular), 0.7 (spinal canal elliptical), 0.6 in the presence of facet compression and 0.5 when compression was made by the disc and facets. Thus, (a / 2) X (b / 2) x $\pi$ (constant) $=$ area [13].

Patients were excluded if they showed the following: diabetes mellitus, hypertension or decompensated heart disease; systemic diseases affecting the lower limbs; neuromuscular diseases; patients with previous surgery on the spine; cognitive deficits that interfere with the ability to understand or interpret the questionnaires; spondylolisthesis, except degenerative; scoliosis with a Cobb angle greater than $10^{\circ}$; hip or knee disorders that could interfere with gait; total or partial arthroplasty in the hip or knee and lumbar canal stenosis in more than three levels.

The IG patients the underwent lumbar decompression surgery using the isolated posterior approach associated with arthrodesis, instrumented with the aid of radioscopy and always by the same senior surgeon. The arthrodesis was stimulated with the placement of a bone graft in the transverse processes and the use of pedicular screw implants without a cage. The surgery was done 1 week after the baseline evaluation. Patients in IG and CG were evaluated 4 weeks after baseline (T4 assessment) and both groups started physical therapy after this evaluation.

In the physical therapy program patients performed 5 exercises. All the exercises were performed in supine position with hips and knees flexed, feet resting on the bed and upper limbs resting along the body. Following the physical therapy program was detailed:

- Exercise 1: After deep inspiration, the patient contracts the muscles of the abdomen and buttocks, making a retroversion for $5 \mathrm{~s}$, relax and restart. Strengthens abdominals and buttocks isometrically, 3 sets of 10 repetitions;

- Exercise 2: Performs gluteus contraction and hip lift (bridge). Strengthens buttocks and stabilizes the lumbar spine, 3 sets of 10 repetitions;

- Exercise 3: Pull one thigh interlacing the hands in the posterior region of the thigh, approaching the knee to the thorax, holding for $30 \mathrm{~s}$ and repeating with the other thigh, alternately performing the movement. Stretches lumbar paravertebral muscles, 10 repetitions on each side;

- Exercise 4: Pull both thighs at the same time, interlacing the hands in the posterior region of the thigh, approaching the knee to the thorax, and holding for $30 \mathrm{~s}$. Stretches lumbar paravertebral muscles, 10 repetitions;

- Exercise 5: Using a stretching strap, hold the strap with both hands, place the strap on the arch of the 
left foot and raise the left foot, straightening the left leg and keeping the right foot on the floor. Hold for $30 \mathrm{~s}$. Stretches hamstring muscles, 10 repetitions on each side.

Patients in both groups took acetaminophen $750 \mathrm{mg}$ up to three times daily for 90 days based on pain and patient's criteria.

Evaluations were performed by a blinded assessor at baseline (T0), after 4 (T4), 12 (T12 - end of physiotherapy), 24 (T24) and 48 (T48) weeks.

Patients were clinically evaluated at each time point to detect possible clinical or surgical complications, such as disabling pain, respiratory and surgical site infection, deep venous thrombosis and vascular complications.

The primary outcome was pain in the last week. This was measured using a visual analogue scale (VAS) ranging from $0 \mathrm{~cm}$ (no pain) to $10 \mathrm{~cm}$ (unbearable pain) [16];

Secondary outcomes were:

- Function - measured using the Roland-Morris questionnaire [17], with scores ranging from 0 to 24 points (lower scores denote better function); the Oswestry questionnaire [18], with scores ranging from 0 to 5 points (lower scores denote better function) and the 6-min walk test (6MWT) following the American Thoracic Society guidelines [19].

- General health. - assessed using the short form 36 (SF-36) questionnaire, with overall scores ranging from 0 to 100, and higher scores denote better general health [20].

- Patient satisfaction with treatment - measured with a Likert scale. The following question was asked: "How do you feel about your treatment?". The patient could select one of the five answer options: 5: much better; 4: a little better; 3: unchanged; 2: a little worse or 1: much worse.

\section{Statistical analysis}

Sample size was calculated for the repeated-measure analysis of variance (ANOVA) with two repetitions per situation, using the VAS for pain as the main parameter, with a standard deviation (SD) of $2 \mathrm{~cm}$. For the determination of a minimal effect of $2 \mathrm{~cm}$, a $5 \% \alpha$ error, $20 \%$ $\beta$ error and SD $(\sigma)$ of $2 \mathrm{~cm}$ were established. The calculation determined a sample of 26 patients per group. Considering a possible loss of 20\%, 63 patients were selected.

The following tests were used in data analysis:

$\checkmark \times 2$, Student's $t$ test and Mann-Whitney were used to determine the homogeneity of the sample at the initial evaluation. $\checkmark$ Repeated-measures ANOVA with Bonferroni adjustments were used to determine differences in the outcomes between groups over time.

$\checkmark$ Pearson correlation were used to determine a correlation between pain and other baseline parameters like age, BMI and canal diameter.

Using intention-to-treat analysis, data from all patients initially enrolled were analyzed. For cases in which there was an interruption of treatment, the patients were first asked to come in and perform only the evaluations. For patients who refused to return for the evaluations, the last data collected were repeated in the subsequent evaluations.

\section{Results}

Sixty-three patients were randomized, 31 patients in the IG and 32 in the CG. The flowchart of the study is presented in Fig. 1.

The mean age of the IG patients was 60.7 years and the CG was 60.2 years. The IG had 8 men and 23 women, the CG had 8 men and 24 women $(p=0.941)$. The demographic data of the sample at the initial evaluation were shown in Table 1. The diameter of the vertebral canal was evaluated at the time of inclusion (Table 1).

During surgery, arthrodeses of different segments were performed. In three patients, one level was set, in eight patients, two levels were set, and in 20 patients, there were three levels.

No significant difference, using repeated measures ANOVA, were found between the groups for pain using the VAS (Table 2, Fig. 2), and in the following domains of SF-36: physical functioning; bodily pain; general health; social functioning; and role emotional and mental health (Table 2).

For function and quality of life, significant difference between groups were found for the following parameters:

- Oswestry $(p=0.006)$ - IG presented worsening in T4 and improvement from T12 to T48 remaining better than CG at the end the study. Significant differences between groups were found at T24 $(p=0.011)$ and T48 ( $p=0.009)$, Table 2 and Fig. 2;

- Roland Morris $(p=0.005)$ - IG presented worsening in T4 and improvement after T12 reaching scores similar to CG in T48. Significant differences between groups were found at T4 $(p=0.001)$ and T12 $(p=0.001)$, Table 2 and Fig. 2;

- 6-min walk test $(p<0.001)$ - IG presented worsening in T4 and improvement after T12 reaching scores similar to CG in T48. Significant differences between the groups were found at T4 $(p=0.001)$ and T12 ( $p=0.024)$, Table 2 and Fig. 2; 


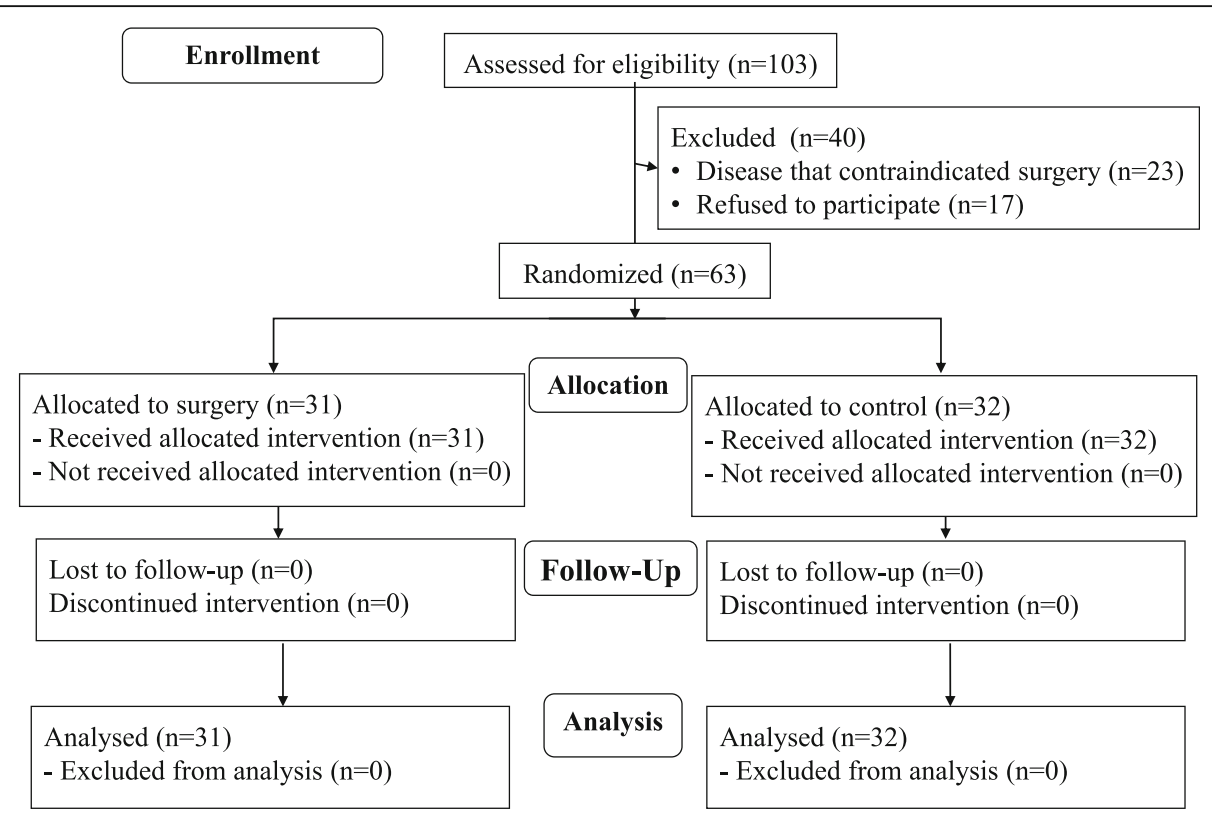

Fig. 1 Study flowchart

- Role physical domain of SF-36 ( $p=0.023)$ - IG presented worsening in $\mathrm{T} 4$ and improvement after T12 reaching scores similar to CG in T48.

Significant differences between groups were found at T4 $(p=0.003)$ and T24 $(p=0.007)$, Table 2 and Fig. 2;

- Vitality domain of SF-36 $(p=0.047)$ - IG presented improvement compared to CG. Significant differences between the groups were found at T4 $(p=0.023)$, T24 $(p=0.014)$ and T48 $(p=0.25)$, Table 2 and Fig. 2.
For treatment satisfaction, a significant difference between the groups was found based on the Likert scale $(p=0.024)$. Analyzing data from the groups separately, we find that at all evaluated times, more patients from the IG choose the "slightly better" alternative: in T4, $80.6 \%$ of the IG versus $21.9 \%$ of the CG chose this option; in T12 83.9\% versus 34.4; in T24.71\% versus 18.8 and in T48 58.1\% versus 31.3 (Table 3 ).

Paracetamol use was similar between groups over time with a mean of $163 \pm 49.88$ tablets for the CG and $174 \pm$ 50.84 for the IG $(p=0.371)$.

Table 1 Demographic data of sample cohorts at baseline

\begin{tabular}{llll}
\hline & $\begin{array}{l}\text { Intervention group } \\
(\boldsymbol{n}=\mathbf{3 1})\end{array}$ & $\begin{array}{l}\text { Control group } \\
(\boldsymbol{n}=\mathbf{3 2})\end{array}$ & $\begin{array}{c}\boldsymbol{P} \\
\text { intergroup }\end{array}$ \\
\hline $\begin{array}{lll}\text { Gender }-\mathrm{n}(\%) \\
\text { Female }\end{array}$ & $23(74.2)$ & $25(78.1)$ & 0.714 \\
$\quad$ Male & $8(25.8)$ & $7(21.9)$ & $60.22(7.27)$ \\
Age - mean (SD) & $60.71(7.41)$ & $163.63(11.45)$ & 0.423 \\
Height - mean (DP) & $161.87(10.06)$ & $71.03(11.79)$ & 0.521 \\
Weight - mean (SD) & $76.74(13.34)$ & $16(50)$ & 0.077 \\
Systemic hypertension - n (\%) & $21(67.7)$ & $9(28.1)$ & 0.151 \\
Diabetes - n (\%) & $6(19.3)$ & & 0.413 \\
Canal diameter in mm ${ }^{2-}-$ mean (SD) & & $100.34(14.19)$ & 0.206 \\
L3/L4 & $94.26(15.31)$ & $81.44(12.63)$ & 0.725 \\
L4/L5 & $81.87(10.64)$ & $86.25(11.28)$ & 0.191 \\
L5/S1 & $84.13(9.29)$ & & \\
\hline
\end{tabular}

Data present as number (percentage) or mean (standard deviation) 


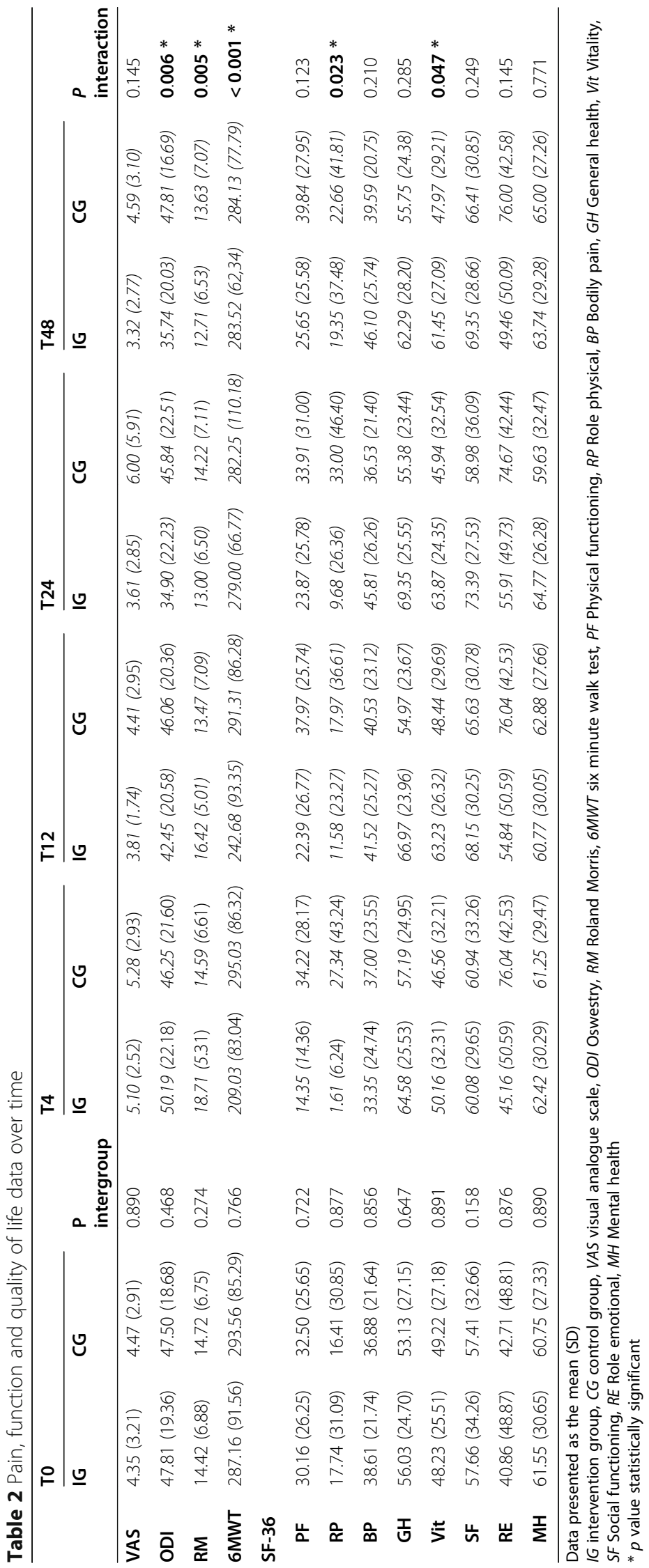




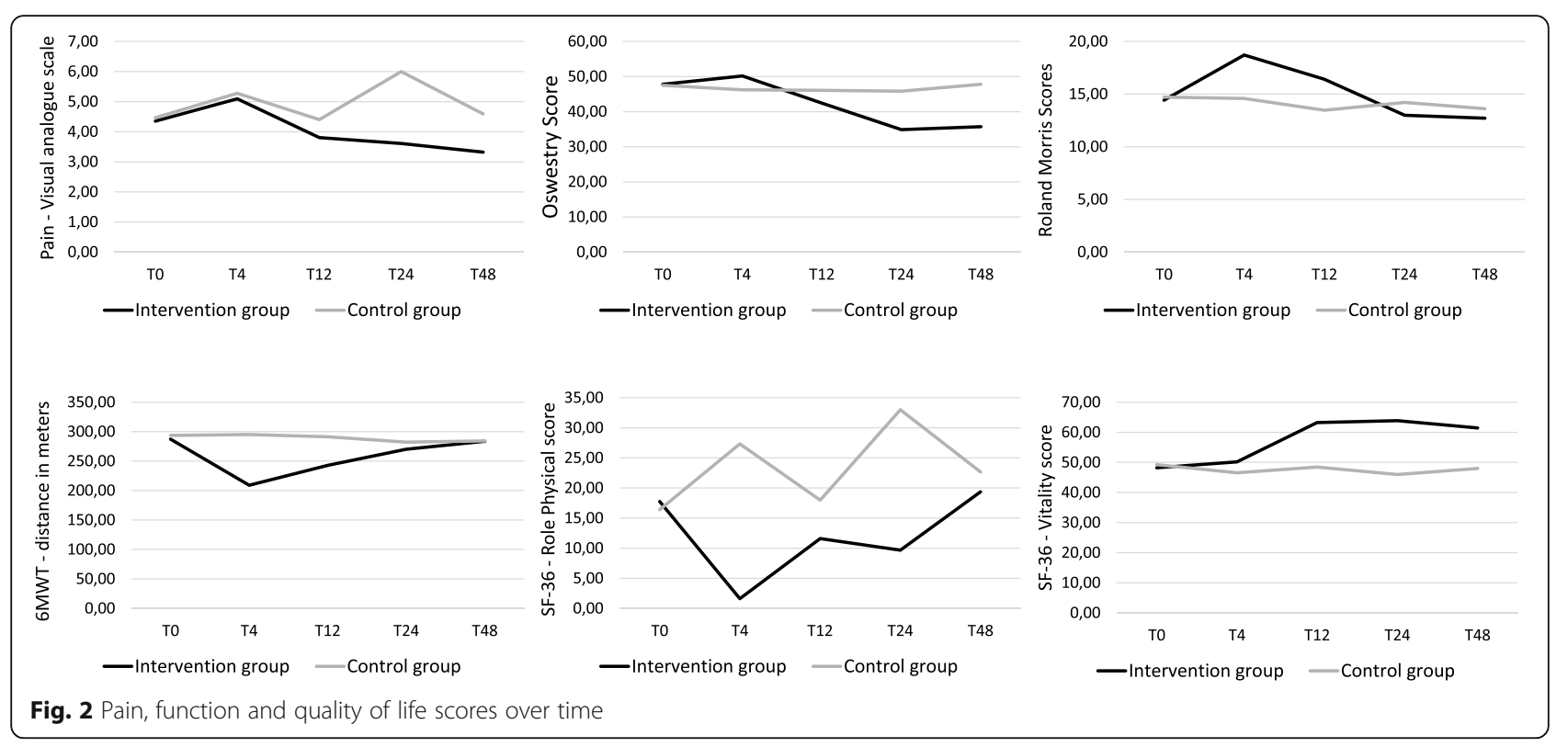

Regarding correlation, using the Pearson test we found low correlation with no statistical evidence between pain and: age $(r=0,066, p=0,605)$, IMC $(r=0,007, p=0,953)$, canal diameter L3/L4 $(r=0,085, p=0,507)$, canal diameter L4/L5 $(r=0,119, p=0,350)$ and canal diameter L5/ $\mathrm{S} 1(r=0,235, p=0,604)$.

\section{Discussion}

The findings of our study show that canal stenosis surgery associated with physical therapy does not improve pain in the short and medium terms, but there is an improvement in function and vitality in the medium term, and patients report satisfaction with the treatment.

In the literature, the vast majority of studies are open and show follow-up data for patients after surgery, with varied surgical techniques used, evaluating whether to perform surgery, the placement of spacers or different laminectomy techniques [7-10]. However, we did not find studies comparing the association of surgical treatment with physiotherapy with physical therapy alone, as in our study.
All of our patients underwent a physiotherapy program created by our team to strengthen the abdominal muscles and stretch the posterior trunk and lower limb muscles because similar exercise programs are performed in daily practice and are indicated for the management of these patients by many authors.

Many outcomes reported in the literature for the evaluation of patients with lumbar spinal stenosis have positive and negative attributes, so a combination of questionnaires or tests may be necessary to properly capture the impact of lumbar stenosis on a patient's pain, disability, and quality of life. As pain is usually the main complaint of lumbar spinal stenosis, we chose this parameter as the main one on this study, and the other questionnaires as Roland Morris, Oswestry, SF-36 and 6 minute walk test were used as secondary outcomes.

It is important to note that in our study, pain worsened in the two groups between the baseline and first reevaluation, and this can be explained by surgery in the IG; however we find it difficult to explain the

Table 3 Patient satisfaction with treatment over time

\begin{tabular}{|c|c|c|c|c|c|c|c|c|c|}
\hline & T4 & & T12 & & T24 & & T48 & & \\
\hline & $\overline{\text { IG }}$ & CG & $\overline{\text { IG }}$ & CG & $\overline{\text { IG }}$ & CG & $\overline{\text { IG }}$ & CG & $\begin{array}{l}P \text { interaction } \\
0.024\end{array}$ \\
\hline Much better & 0 & $1(3.1)$ & $2(6.5)$ & $4(12.5)$ & $3(9.7)$ & $2(6.3)$ & $7(22.6)$ & $6(18.8)$ & \\
\hline Slightly better & 25 (80.6) & $7(21.9)$ & $26(83.9)$ & $11(34.4)$ & $22(71)$ & $6(18.8)$ & $18(58.1)$ & $10(31.3)$ & \\
\hline Unchanged & $6(19.4)$ & $12(37.5)$ & $2(6.5)$ & $8(25)$ & $3(9.7)$ & $13(40.6)$ & $5(16.1)$ & $7(21.9)$ & \\
\hline Slightly worse & 0 & $11(34.4)$ & $1(3.2)$ & $9(28.1)$ & $2(6.3)$ & $10(31.1)$ & $1(3.2)$ & $9(28.1)$ & \\
\hline Much worse & 0 & $1(3.1)$ & 0 & 0 & $1(3.2)$ & $1(3.1)$ & 0 & 0 & \\
\hline
\end{tabular}

Data presented as $\mathrm{n}(\%)$; IG intervention group, CG control group; data presented as $\mathrm{n}(\%)$ 
same behavior in the CG because the patients had not yet begun physical therapy.

During the physiotherapy period, both groups improved in pain, and after the end of the physiotherapy, the patients of the IG continued to improve, with an improvement of approximately $1 \mathrm{~cm}$ in VAS for pain. By contrast, the CG presented a worsening in the 3 months after the end of the physiotherapy, followed by an improvement until the end of the study. Despite these small differences between groups, we found no statistically or clinically significant difference between the groups over time for the pain parameter.

Other studies showed mixed results regarding pain measured by the VAS. Ulrich et al., described improvement at 6 months, maintained for 12 months, and showing a more stable evolution, but his work was in older patients and without a control group [21]. Skolasky et al., showed that after 6 months, surgical patients had an improvement of pain with a $2 \mathrm{~cm}$ drop in VAS. This drop remained during the follow-up of 12 months, with $63 \%$ of his patients improving more than $2 \mathrm{~cm}$. After 1 year, patients were better than baseline in both groups [22]. Ahmad et al. compared two surgical techniques, and also showed an improvement of $2 \mathrm{~cm}$ in VAS [23]. In assessing clinically treated patients, Schneider et al. showed that the VAS of the patients did not change, similar to our study [24].

For function, we found significant differences in the three instruments used to measure it. Oswestry showed a statistically and clinically significant improvement in favor of IG. In T24, the improvement was 12.9 points and in T48 it was 12.07 points. Some authors consider 12.8 points as a clinically important difference for the patient with low back pain $[25,26]$.

In other studies, superior improvements were observed. Ahmad et al. found an improvement of 23 points in the Oswestry in the operated patients, despite their study cohort [23]. Hemansen et al. compared three different decompression techniques, finding an improvement of approximately 20 points in Oswestry, regardless of the technique used [27]. Skolasky et al. did a prospective, but not controlled, analysis for a period of 12 months and observed improvement in the Oswestry at 3 months postoperative, maintaining the values at 6 months and with an improvement of 20 points in the final evaluation [22].

For function with both the Roland Morris questionnaire and the 6MWT questionnaire, we found a difference between the groups over time, but this was due to a worsening of the GI in T4, shortly after surgery and before starting physical therapy, and then both parameters were improved. At the end of the study, there were no differences between both groups. Again, worsening in the IG can be explained by the surgical intervention, which did not occur in the CG.
Compared with other studies, Ulricha et al., in a prospective multicenter cohort study, evaluated Roland Morris in patients undergoing surgery with a 1 year follow-up. In this period, he observed that there was a drop of 7 points in the questionnaire, with one difference being the average age at work of 82 years [21].

Regarding 6MWT, Försth et al., in an randomized controlled trial (RCT), obtained divergent results after fusion surgery because their patients showed an improvement of more than $100 \mathrm{~m}$ after 12 months of surgery [28]. Yamashita et al., in 2003, followed patients after laminectomy surgery in an open-label study and demonstrated that operated patients had no improvement in the ability to ambulate after surgery [29]. Slätis et al. showed in an RCT that the ambulatory or control patients' ambulatory ability did not change after 1 year of surgery, consistent with the results of this research [30]. Malmivaara et al., in an RCT comparing surgical and non-surgical treatment, showed that after 2 years of follow-up, their patients who underwent surgical treatment were better at pain and limitations, but their ability to walk remained unchanged [31]. Rodrigues et al., in an RCT comparing the use of oral corticosteroids with placebo in patients with canal stenosis, also showed that after 6 months of evolution, no improvement was observed in both groups and that the placebo group had, on average, an increase of only $10 \mathrm{~m}$ in relation to the initial evaluation [32].

In the quality of life questionnaire, the differences found in role physical fluctuated, with improvement and worsening of both groups over time, but with no difference between them at the end of the study. By contrast, vitality showed an improvement of approximately $15 \%$ in the GI in T12, T24 and T48 $(p=0.023, p=0.014$ and $p=0.25$, respectively) comped with the control group, which may also have influenced the improvement of function and satisfaction with treatment.

Westein et al. performed an RCT comparing surgical and non-surgical treatment in patients with canal stenosis and did not detect improvement in the SF-36 questionnaire, as well as in the other evaluation instruments used [33]. In another RCT comparing surgical and nonsurgical treatment in patients with degenerative spondylolisthesis, Westein et al. described positive results with surgery in the pain and physical function domains in the SF-36, different from that observed in our study [34].

The transient worsening of the operated group in some variables could be explained by the expected limitations and pain in the postoperative period, without, however, being perpetuated. These, as well as other results, demonstrate the safety of the surgery because the patients were not worse than before and there were no clinical complications resulting from the surgery.

Patient satisfaction, measured with the Likert scale, showed an advantage for those operated on. The 
functional improvement experienced by these patients may have generated the perception of more satisfaction with the treatment, despite not having less pain. It is impossible to blind patients, which may also explain this difference because operated patients would tend to value a major medical intervention with all of the additional care that they naturally received in relation to the CG.

El-Abed et al. showed in their case-control study that patients who underwent surgery significantly improved their evaluation [35]. Many studies using the Likert-type scale as an evaluation tool were not identified. In summary, the operated patients were more satisfied with the treatment than the control group.

The surgery performed in all patients of this study is a lumbar decompression using a posterior approach associated with arthrodesis. As our patients were relatively young, if we performed only decompression surgery without arthrodesis, we would probably have many patients with instability. Thus, we chose to perform arthrodesis after decompression to unify the procedure to all patients, once only older patients with a lot of arthrosis in the spine would not be left with an unstable spine without instrumented arthrodesis. So, it is important to say that our results do not be generalized to patients undergoing decompression surgery without arthrodesis.

The selection of patients may influence the results; however, we selected patients who are usually submitted for this type of surgery based on internationally accepted inclusion criteria. Other population, with different diseases duration, degree of disability, impairment of canal stenosis, or another age group, deserve further studies. Another limitation of our study is related to the disease duration, patients were included if have chronic pain (more than 6 months), but we do not ask about disease duration in the baseline. A longer duration of the disease usually leads to more disabilities and is an important parameter that may have influenced the results of this study.

\section{Conclusion}

In conclusion, lumbar stenosis surgery had no effect on pain improvement in the short and medium terms. Function and vitality were better in the group that underwent surgery in the medium term, and patients were more satisfied with the surgical treatment. Further studies should be performed, and surgery should be indicated with caution in patients like ours.

\section{Clinical messages}

- Patients who underwent surgery for lumbar canal stenosis associated with physical therapy for 12 weeks and followed by 1 year showed no improvement in pain when compared to the control group who did only physical therapy;
- The function, vitality and satisfaction with the treatment were better in patients undergoing surgery in the medium term.

- Lumbar stenosis surgery had no effect on pain in short and medium term.

\section{Abbreviations}

IG: Intervention group; CG: Control group; VAS: Visual analogue scale; 6MWT: 6-min walk test; SF-36: Short form 36; ANOVA: Analysis of variance; SD: Standard deviation; RCT: Randomized controlled trial

\section{Acknowledgements}

Not applicable.

\section{Authors' contributions}

Rodrigues LCL - Conceptualization, Methodology, Data collection, WritingOriginal draft preparation and Editing; Jamil Natour - Conceptualization, Methodology, writing-reviewing and editing. The author(s) read and approved the final manuscript.

\section{Funding}

This study did not receive financial resources.

Availability of data and materials

Study data can be made available upon reasonable request to the principal investigator.

\section{Declarations}

Ethics approval and consent to participate

This study was approved by the ethics committee (CAAE:

16125013.6.0000.0066) and all participants gave their informed consent

before participation.

Consent for publication

Not applicable.

Competing interests

None to declare.

\section{Author details}

${ }^{1}$ Rheumatology Division, Universidade Federal de São Paulo - Escola Paulista de Medicina (Unifesp-EPM), Sao Paulo, SP 04023900, Brazil. ${ }^{2}$ Faculdade de Medicina Santa Marcelina, Sao Paulo, Brazil.

Received: 26 October 2020 Accepted: 27 April 2021

Published online: 12 May 2021

References

1. Verbiest $\mathrm{H}$. A radicular syndrome from developmental narrowing of the lumbar vertebral canal. J Bone Joint Surg (Br). 1954;36:230-7.

2. Verbiest $H$. The significance and principles of computerized axial tomography in idiopathic developmental stenosis of the bony lumbar vertebral canal. Spine. 1979;4(4):369-78. https://doi.org/10.1097/00007632-1 97907000-00005.

3. Naylor A. Factors in the development of the spinal stenosis syndrome. J Bone Joint Surg (Br). 1978;61:319-28.

4. Thomas SA. Spinal stenosis: history and physical examination. Phys Med Rehabil Clin N Am. 2003;14(1):29-9. https://doi.org/10.1016/S1047-9651(02 )00049-9.

5. Blau JN, Lond MB. Intermittent claudication of the cauda equina. Lancet. 1961;277(7186):1081-6. https://doi.org/10.1016/S0140-6736(61)92310-8.

6. Deyo RA, Gray DT, Kreuter W, Mirza S, Martin B. United States trends in lumbar fusion surgery for degenerative conditions. Spine. 2005;30(12):14415. https://doi.org/10.1097/01.brs.0000166503.37969.8a.

7. Shabat S, Arizon Z, Folman Y, Leitner J, David R, Pevzner E, et al. Long-term outcomes of decompressive surgery for lumbar spinal stenosis in octogenarians. Eur Spine J. 2008;17(2):193-8. https://doi.org/10.1007/s00586007-0514-8. 
8. Airaksinen $\mathrm{O}$, Hermo A, Saari T. Surgical treatment of lumbar spinal stenosis: patients' postoperative disability and working capacity. Eur Spine J. 1994; 3(5):261-4. https://doi.org/10.1007/BF02226576.

9. Hurri $H$, Slätis $P$, Soini J, Tallroth $K$, Alaranta H, Laine $T$, et al. Lumbar spinal stenosis: assessment of long-term outcome 12 years after operative and conservative treatment. J Spinal Disord. 1998;11(2):110-5.

10. Atlas S, Keller RB, Robson D, Deyo RA, Singer DE. Surgical and nonsurgical management of lumbar spinal stenosis: four-years outcomes from the Maine lumbar spine study. Spine. 2000;25(5):556-62. https://doi.org/10.1097/ 00007632-200003010-00005.

11. Atlas SJ, Keller RB, Wu YA, Deyo RA, Singer DE. Long-term outcomes of surgical and nonsurgical management of lumbar spine study. Spine. 2005; 30(8):936-43. https://doi.org/10.1097/01.brs.0000158953.57966.c0.

12. Arbit E, Pannullo S. Lumbar stenosis: a clinical review. Clin Orthop Relat Res. 2001;384:137-43. https://doi.org/10.1097/00003086-200103000-00016.

13. Hamanishi C, Matukura N, Fujita M, Tomihara M, Tanaka S. Cross-sectional area of the stenotic lumbar dural tube measured from the transverse views of magnetic resonance imaging. J Spinal Disord. 1994;7(5):388-93.

14. Madsen R, Jensen TS, Pope M, Sørensen JS, Bendix T. The effect of body position and axial load on spinal canal morphology: an MRI study of central spinal stenosis. Spine. 2008;33:61-7.

15. Lee CK, Rauschning W, Glenn W. Lateral lumbar spinal canal stenosis: classification, pathologic anatomy and surgical decompression. Spine. 1988; 13:313-20.

16. Zanoli G. Outcome assessment in lumbar spine surgery. Acta Orthop Suppl. 2005;76(318):5-47.

17. Nusbaum L, Natour J, Ferraz MB, Goldenberg J. Translation, adaptation and validation of the Roland-Morris questionnaire-Brazil Roland-Morris. Braz J Med Biol Res. 2001;34(2):203-10. https://doi.org/10.1590/S0100-879X2001 000200007.

18. Vigatto R, Alexandre NM, Correa Filho HR. Development of a Brazilian Portuguese version of the Oswestry Disability Index: cross-cultural adaptation, reliability, and validity. Spine. 2007;32:481-6.

19. ATS Committee on Proficiency Standards for Clinical Pulmonary Function Laboratories. ATS statement: guidelines for the six-minute walk test. Am J Respir Crit Care Med. 2002;166:111-7.

20. Ciconelli RM, Ferraz MB, Santos W, Meinão I, Quaresma MR. Tradução Para Língua Portuguesa E Tradução Do Questionário Genérico De Avaliação De Qualidade De Vida SF-36 (Brasil SF-36). Rev Bras Reumatol. 1999;39:143-50.

21. Ulrich NH, Kleinstück F, Woernle CM, Antoniadis A, Winklofer S, Burgstaller $J M$, et al. Clinical outcome in lumbar decompression surgery for spinal canal stenosis in the aged population: a prospective Swiss multicenter cohort study. Spine. 2015;40:415-22.

22. Skolasky RL, Wegener ST, Maggard AM, Riley LH 3rd. The impact of reduction of pain after lumbar spine surgery: the relationship between changes in pain and physical function and disability. Spine. 2014;39:1426-32.

23. Ahmad S, Hamad A, Bhalla A, Turner S, Balain B, Jaffray D. The outcome of decompression alone for lumbar spinal stenosis with degenerative spondylolisthesis. Eur Spine J. 2017;26(2):414-9. https://doi.org/10.1007/ s00586-016-4637-7

24. Schneider MJ, Terhorst L, Murphy D, Stevans JM, Hoffman R, Cambron JS. Exploratory analysis of clinical predictors of outcomes of nonsurgical treatment in patients with lumbar spinal stenosis. J Manip Physiol Ther. 2016;39(2):88-94. https://doi.org/10.1016/j.jmpt.2016.01.001.

25. Johnsen LG, Hellum C, Nygaard OP, Storheim K, Brox Jl, Rossvoll I, et al. Comparison of the SF6D, the EQ 5D, and the Oswestry disability index in patients with chronic low back pain and degenerative disc disease. BMC Musculoskelet Disord. 2013;14(1):148. https://doi.org/10.1186/1471-24 74-14-148.

26. Copay AG, Glassman SD, Subach BR, Berven S, Schuler TC, Carreon LY. Minimum clinically important difference in lumbar spine surgery patients: a choice of methods using the Oswestry disability index, medical outcomes study questionnaire short form 36, and pain scales. Spine J. 2008:8(6):96874. https://doi.org/10.1016/.spinee.2007.11.006.

27. Hermansen E, Romild UK, Austevoll IM, Solberg T, Storheim K, Brox Jl, et al. Does surgical technique influence clinical outcome after lumbar spinal stenosis decompression? A comparative effectiveness study from the Norwegian registry for spine surgery. Eur Spine J. 2017;26(2):420-7. https:// doi.org/10.1007/s00586-016-4643-9.
28. Försth $\mathrm{P}$, Ólafsson $\mathrm{G}$, Carlsson T. A randomized, controlled trial of fusion surgery for lumbar spinal stenosis. N Engl J Med. 2016;374(15):1413-23. https://doi.org/10.1056/NEJMoa1513721.

29. Yamashita K, Hayashi J, Ohzono K, Hiroshima K. Correlation of patient satisfaction with symptom severity and walking ability after surgical treatment for degenerative lumbar spinal stenosis. Spine. 2003;28:2477-81.

30. Slätis P, Malmivaara A, Heliövaara M, Sainio P, Herno A, Kankare J, et al. Long-term results of surgery for lumbar spinal stenosis: a randomised controlled trial. Eur Spine J. 2011;20(7):1174-81. https://doi.org/10.1007/ s00586-010-1652-y.

31. Malmivaara A, Slätis P, Heliövaara M, Sainio P, Kinnunen H, Kankare J, et al. Surgical or nonoperative treatment for lumbar spinal stenosis? A randomized controlled trial. Spine. 2007:32:1-8.

32. Rodrigues LC, Natour J. A double-blind, randomized controlled, prospective trial assessing the effectiveness of oral corticoids in the treatment of symptomatic lumbar canal stenosis. J Negat Results Bioemed. 2014;13(1):13. https://doi.org/10.1186/1477-5751-13-13.

33. Weinstein JN, Tosteson TD, Lurie JD, Tosteson ANA, Blood E, Hanscom B, et al. Surgical versus nonsurgical therapy for lumbar spinal stenosis. N Engl J Med. 2008;358(8):794-10. https://doi.org/10.1056/NEJMoa0707136.

34. Weinstein JN, Lurie JD, Tosteson TD, Hanscom B, Tosteson ANA, Blood EA, et al. Surgical versus nonsurgical treatment for lumbar degenerative spondylolisthesis. N Engl J Med. 2007;356(22):2257-70. https://doi.org/10.1 056/NEJMoa070302.

35. El-Abed K, Barakat M, Ainscow D. Multilevel lumbar spinal stenosis decompression: midterm outcome using a modified hinge osteotomy technique. J Spinal Disord Tech. 2011;24(6):376-80. https://doi.org/10.1097/ BSD.0b013e3182014aa1.

\section{Publisher's Note}

Springer Nature remains neutral with regard to jurisdictional claims in published maps and institutional affiliations.

Ready to submit your research? Choose BMC and benefit from:

- fast, convenient online submission

- thorough peer review by experienced researchers in your field

- rapid publication on acceptance

- support for research data, including large and complex data types

- gold Open Access which fosters wider collaboration and increased citations

- maximum visibility for your research: over $100 \mathrm{M}$ website views per year

At $\mathrm{BMC}$, research is always in progress.

Learn more biomedcentral.com/submissions 